\title{
Hipofisitis autoinmune como causa de diabetes insípida central completa e hipogonadismo hipogonadotrófico en una mujer joven: revisión de un caso
}

\section{Autoimmune hypophysitis as a cause of complete central diabetes insipidus and hypogonadotrophic hypogonadism in a young woman: A case review}

\author{
Sara M. Cervantes-Urenda, Stefania Petarra-del Río, Alejandro Quintanilia-Leyva, Miguel A Polanco-Preza*, \\ Salvador Plascencia-PÉrez y José R. Barrientos-Ávalos
}

Servicio de Endocrinología, Hospital Civil de Guadalajara Fray Antonio Alcalde, Universidad de Guadalajara, Guadalajara, Jalisco, México

RESUMEN

La hipofisitis autoinmune es una enfermedad rara, definida por la inflamación de la glándula hipófisis debido a diversos mecanismos autoinmunitarios, y que puede ser asintomática o manifestarse en forma de deficiencias hormonales. Presentamos el caso de una mujer joven que fue derivada a nuestra unidad por poliuria y polidipsia de un año de evolución, cefalea bilateral constante que mejoraba, pero no cedía, a analgésicos, acompañada de náuseas y miodesopsias. Tras realizar pruebas de laboratorio y de imagen, fue diagnosticada con hipofisitis autoinmune que ocasionó diabetes insípida central completa e hipogonadismo hipogonadotrófico.

Palabras clave: Hipofisitis autoinmune. Diabetes insípida. Hipogonadismo hipogonadotrófico. Poliuria. Polidipsia.

\begin{abstract}
Autoimmune hypophysitis is a rare disease, defined by inflammation of the pituitary gland due to some autoimmune mechanism. It can be asymptomatic or manifest in the form of hormonal deficiencies. We present the case of a young woman who was referred to our unit for polyuria and polydipsia of a year of evolution, constant bilateral headache, accompanied by nausea and myodesopsias, which improved, but did not disappear with analgesics. After performing laboratory and imaging tests, she was diagnosed with autoimmune hypophysitis causing complete central diabetes insipidus and hypogonadotrophic hypogonadism.
\end{abstract}

Key words: Autoimmune hypophysitis. Diabetes insipidus. Hypogonadotrophic hypogonadism. Polyuria. Polydipsia.
Correspondencia:

*Miguel A. Polanco-Preza

E-mail: mpolancopreza@yahoo.com.mx
Fecha de recepción: 26-06-2021

Fecha de aceptación: 20-10-2021

DOI: 10.24875/RME.M21000018
Disponible en internet: 18-11-2021

RevMexEndocrinol Metab Nutr.2021;8:(SUPL.2):19-23

2462-4144 / @ 2021 Sociedad Mexicana de Nutrición y Endocrinología, AC. Publicado por Permanyer. Este es un artículo open access bajo la licencia CC BY-NC-ND (http://creativecommons.org/licenses/by-nc-nd/4.0/). 


\section{INTRODUCCIÓN}

La hipofisitis es una afección rara que abarca un amplio espectro de lesiones inflamatorias que pueden afectar a la hipófisis y el tallo hipofisario.

La hipofisitis autoinmunitaria es la forma más frecuente y debido a su amplio espectro de presentación, su identificación supone un reto clínico importante y obliga a considerar diversas entidades clínicas dentro del diagnóstico diferencial ${ }^{1}$.

Es indispensable un abordaje sistemático, que incluya la realización de pruebas de imagen al inicio y durante el seguimiento. En general, el pronóstico es bueno, y se ha descrito resolución espontánea en algunos casos, aunque puede persistir algún déficit funcional hipofisario de manera permanente ${ }^{1}$.

\section{REPORTE DEL CASO}

Paciente de sexo femenino de 19 años, que consultó por poliuria (10 veces/día) y polidipsia (3 I/día) de un año de evolución, cefalea bilateral constante (escala visual analógica 8/10), que mejoraba, pero no cedía, a analgésicos, acompañada de náuseas y miodesopsias. Desde hace ocho meses la paciente presentó amenorrea secundaria y pérdida de peso de aproximadamente $10 \mathrm{~kg}$. En los meses previos a nuestra valoración, aumentó la polidipsia hasta 15 L/día y la poliuria hasta 15 veces/día y se agregó nicturia (5 veces/noche), motivos por los que fue derivada a valoración por el servicio de endocrinología. Persistía la cefalea, de carácter intermitente y de intensidad leve, astenia, adinamia y piel seca.

Entre los antecedentes familiares, su madre padece hipertensión arterial sistémica (HAS) y su padre prediabetes e HAS. El abuelo paterno había fallecido por infarto de miocardio a los 70 años de edad. La paciente negó antecedentes patológicos y no consumía ningún medicamento. Presentó la menarca a los 15 años y refería inicialmente ciclos menstruales irregulares (ciclos $60 \times 3$ ) con oligomenorrea y posteriormente amenorrea.
En la exploración física no se observaron datos clínicos relevantes y los signos vitales estaban dentro de los parámetros normales.

Entre los diagnósticos diferenciales contemplados se encontraban diabetes insípida central, poliuria primaria, diuresis osmótica y diabetes insípida nefrogénica.

Se solicitaron pruebas de electrolitos, que mostraban valores normales, igual que la glucosa, urea y creatinina. La osmolaridad plasmática también era normal (297 mOsm/L). No se encontraron alteraciones en la biometría hemática ni en las pruebas de función hepática. En el examen general de orina se reportaron una densidad de $1,003 \mathrm{~g} / \mathrm{mL}$ y el resto era normal. Los electrolitos urinarios eran: sodio 16 $\mathrm{mEq} / \mathrm{l}$, potasio $16 \mathrm{mEq} / \mathrm{L}$ y urea $340.7 \mathrm{mg} / \mathrm{L}$. Con base en ello se calculó la osmolaridad urinaria, que fue de $122 \mathrm{mOsm} / \mathrm{kg}$. Estas pruebas permitieron determinar que la paciente tenía poliuria hipotónica, por lo que se realizó el test de privación de agua. La paciente inició con un peso de 63.2 kg y se midió la osmolaridad urinaria cada hora $\mathrm{y}$, a las 6 horas, la paciente tuvo un peso de $61.2 \mathrm{~kg}$, sin cambios en la osmolaridad urinaria $(70 \mathrm{mOsm} / \mathrm{kg})$. Al ser la pérdida de peso superior al $3 \%$ del peso corporal, se procedió a la administración de $2 \mu \mathrm{g}$ de desmopresina IV y a las 2 horas, la paciente tuvo una osmolaridad urinaria de $875 \mathrm{mOsm} / \mathrm{kg}$, y se observó una disminución significativa de la uresis. El aumento superior al $50 \%$ de la osmolaridad urinaria hizo sospechar la presencia de diabetes insípida central. La copeptina basal fue de $0.93 \mathrm{pmol} / \mathrm{L}$ y tras la restricción hídrica de $0.91 \mathrm{pmol} / \mathrm{L}(<2.6)$, lo que descartó una diabetes insípida nefrogénica y orientó hacia una diabetes insípida central ${ }^{2,3}$.

Se realizó una resonancia magnética (RM) hipofisaria y las imágenes en T1 no mostraban evidencia de tumor hipofisario o hipotalámico, ni silla turca vacía. Las imágenes contrastadas con gadolinio mostraban un realce intenso y homogéneo de la hipófisis y un engrosamiento del tallo hipofisario, con una medición > $3.5 \mathrm{~mm}$, que son datos sugestivos de una hipofisitis autoinmune (Fig. 1).

Se solicitaron pruebas para determinar el resto del perfil hipofisiario con el fin de conocer si existía el compromiso de otro eje, y se observó un nivel bajo de estradiol $(8.1 \mathrm{pg} / \mathrm{mL})$, con hormona foliculoestimulante 

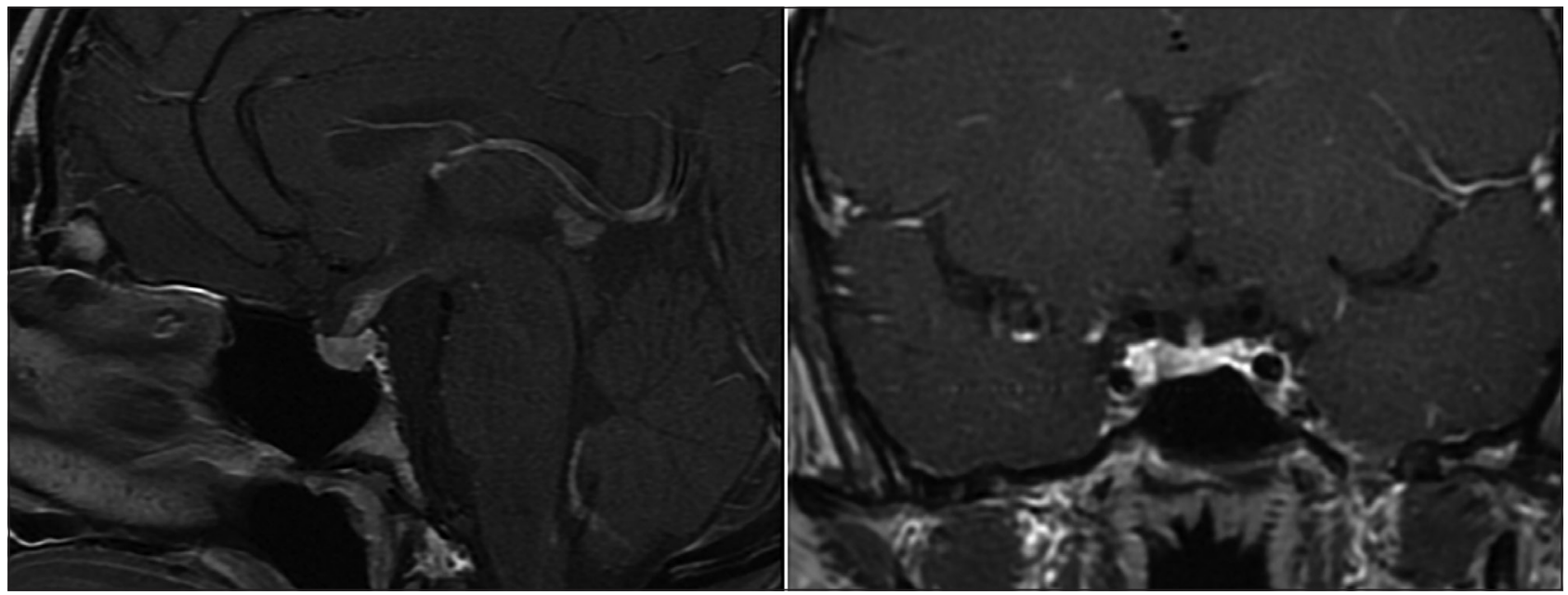

Figura 1. Imágenes realzadas con gadolinio que muestran un realce intenso y homogéneo de la hipófisis y un engrosamiento del tallo hipofisario.
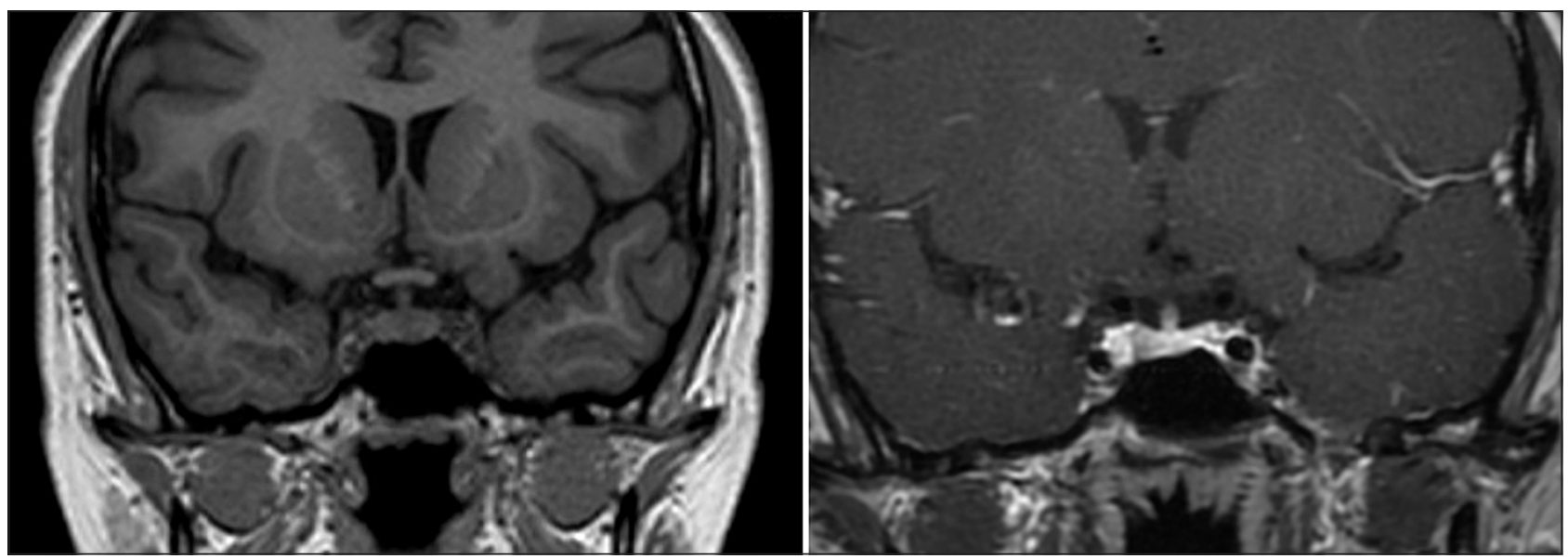

Figura 2. Las imágenes a los 10 meses de seguimiento siguen mostrando engrosamiento del tallo hipofisiario, sin progresión.

y hormona luteinizante sin alteraciones y el resto de los parámetros en rango normal para edad y sexo. Los anticuerpos antiperoxidasa, que se solicitaron por la relación entre la hipofisitis autoinmune y la tiroiditis de Hashimoto, fueron negativos $(<10 \mathrm{Ul} / \mathrm{mL})$.

La primera impresión diagnóstica fue una hipofisitis autoinmune que causó diabetes insípida central completa e hipogonadismo hipogonadotrófico.

Se decidió un manejo conservador, iniciando tratamiento con tabletas de desmopresina $0.1 \mathrm{mg}$ cada $12 \mathrm{~h}$ y etinilestradiol/levonorgestrel $0.03 / 0.15 \mathrm{mg}$.

En el seguimiento clínico a los 10 meses, la paciente negó polidipsia o poliuria, refirió recuperación de peso $(69 \mathrm{~kg})$ y ciclos menstruales regulares. Se solicitaron pruebas de perfil hipofisiario con resultados normales. La RM continúo mostrando engrosamiento del tallo hipofisiario, sin progresión (Fig. 2).

\section{DISCUSIÓN}

La hipofisitis autoinmune es la forma más frecuente de hipofisitis y se define como cualquier inflamación selar o supraselar que condicione cambios estructurales en la región hipotálamo-hipofisiaria y distintos grados de deficiencias hormonales. Aunque antes se le atribuía un componente autoinmune predominante para su presentación, actualmente se considera una 
entidad heterogénea, causada por trastornos autoinmunes mayoritariamente, pero también por procesos inflamatorios o neoplásicos o incluso por algunos fármacos (medicamentos antineoplásicos) ${ }^{1}$.

Desde el punto de vista etiológico, puede clasificarse como primaria o secundaria (Tabla 1), y en función de su localización, como adenohipofisitis linfocítica, infundíbulo-neurohipofisitis linfocítica y parahipofisitis linfocítica.

La incidencia es de 9 casos por cada 1,000,000 pacientes/año, aunque probablemente está subestimada porque las cifras están proyectadas a partir de la experiencia de un único centro en Reino Unido. Mientras que Japón y EE.UU. son los países donde se han reportado incidencias más elevadas (27 y $19 \%$, respectivamente) ${ }^{1}$.

Según un estudio alemán, la mitad de los casos inician con diabetes insípida y solo un $11 \%$ se asocia con el embarazo. La deficiencia hormonal más frecuente es el hipogonadismo hipogonadotrófico, seguido por hormona de crecimiento, y el hallazgo radiológico más frecuente es el engrosamiento del tallo hipofisiario ${ }^{4}$.

Los resultados de una serie mexicana de nueve pacientes son similares, aunque la localización más frecuente fue una infundíbulo-neurohipofisitis ${ }^{5}$.

La hipofisitis linfocítica es la causa de hasta el 70\% de las hipofisitis, y el $50 \%$ de los pacientes presentan otra endocrinopatía o enfermedad sistémica autoinmune asociada? .

La historia natural de la enfermedad es similar a otras enfermedades autoinmunes y se caracteriza por episodios de remisión y recaída, aunque también puede haber una progresión hacia un estado crónico, en relación con el daño celular del hipotálamo-hipófisis y la producción de citocinas a nivel local ${ }^{1}$.

La histopatología varía según el tipo de hipofisitis primaria, aunque suele encontrarse infiltración linfocítica, y los linfocitos pueden agruparse en folículos linfoides con centros germinales. Se pueden acompañar de células plasmáticas y culmina con una fibrosis extensa ${ }^{1}$.

La presentación clínica podría agruparse en cuatro fenotipos: a) con predominio del efecto masa, con cefalea intensa y trastornos visuales; b) déficit
Tabla 1. Clasificación etiológica de la hipofisitis autoinmune

\begin{tabular}{ll}
\hline Primaria & Secundaria \\
\hline - Linfocítica & - Endocrinopatías \\
- Granulomatosa & autoinmunes \\
- Xantomatosa & - Enfermedades sistémicas \\
- Necrotizante & autoinmunes \\
- Mediada por lgG4 & - Vasculitis \\
& - Trastornos inflamatorios/ \\
& proliferativos \\
& - Neoplasias de la región selar \\
& y supraselar \\
& - Inducida por fármacos \\
& - Infecciones \\
& - Síndromes paraneoplásicos \\
\hline
\end{tabular}

Ig: inmunoglobulina.

hormonal (hipocortisolismo, hipogonadismo, hipotiroidismo); c) diabetes insípida, con poliuria y polidipsia, y d) hiperprolactinemia.

En la fase aguda o subaguda, algunos pacientes son asintomáticos; el resto presenta signos y síntomas de efecto masa (cefalea, defectos visuales, náuseas y vómitos), así como alteraciones hormonales, principalmente secundarias a deficiencia de hormona adrenocorticotropa, hormona estimulante del tiroides, gonadotropinas y prolactina. El paciente puede progresar a la fase crónica, donde hay una fibrosis y atrofia, acompañada de deficiencias monohormonales o plurihormonales.

En nuestra paciente, los síntomas principales fueron la poliuria y la polidipsia, aunque también presentó cefalea y náuseas. Para iniciar el abordaje, el síntoma pivote fue la poliuria.

Para el abordaje clínico es fundamental una historia clínica y exploración física minuciosas, así como una evaluación bioquímica para conocer la función hipofisiaria y de los tejidos diana. Si bien la biopsia hipofisiaria es el gold standard para el diagnóstico, se realiza en pocos casos, por lo que la RM es crucial para ayudar al diagnóstico y descartar otras causas.

El score de Gutenberg se utiliza para establecer la diferenciación entre un adenoma hipofisiario no funcional vs. una hipofisitis autoinmune. Un puntaje $\geq 1$ es sugestivo de un adenoma y un puntaje $\leq 0$ se asocia a hipofisitis linfocítica ${ }^{6}$. 
Las características que sugieren hipofisitis autoinmune incluyen un crecimiento hipofisiario homogéneo, extensión supraselar simétrica, engrosamiento del tallo hipofisiario sin desplazamiento, respaldado además por reforzamiento temprano, homogéneo e intenso al administrar contraste y ausencia de hiperintensidad de la neurohipófisis en las imágenes ponderadas en T1, particularmente en pacientes que presentan diabetes insípida ${ }^{7}$. Los avances en el desarrollo de técnicas de imagen no invasivas han contribuido al incremento de los casos diagnosticados.

El abordaje terapéutico se basa en el seguimiento del paciente asintomático sin extensión extraselar o hipocortisolismo importante. En pacientes con síntomas intensos pueden administrarse principalmente glucocorticoides, aunque no hay una postura clara sobre las opciones terapéuticas de esta entidad. En fase crónica está indicada la terapia de reemplazo hormonal ${ }^{8}$.

En conclusión, el diagnóstico de la hipofisitis autoinmune supone un reto clínico, ya que la forma de presentación es muy variada, la historia natural no es predecible y puede presentar numerosos cambios clínicos, morfológicos y funcionales.

\section{FINANCIAMIENTO}

La presente investigación no ha recibido ninguna beca específica de agencias de los sectores públicos, comercial o con ánimo de lucro.

\section{CONFLICTO DE INTERESES}

Los autores declaran no tener conflicto de intereses.

\section{RESPONSABILIDADES ÉTICAS}

Protección de personas y animales. Los autores declaran que para esta investigación no se han realizado experimentos en seres humanos ni en animales.

Confidencialidad de los datos. Los autores declaran que han seguido los protocolos de su centro de trabajo sobre la publicación de datos de pacientes.

Derecho a la privacidad y consentimiento informado. Los autores han obtenido el consentimiento informado de los pacientes y/o sujetos referidos en el artículo. Este documento obra en poder del autor de correspondencia.

\section{BIBLIOGRAFÍA}

1. Gubbi S, Hannah-Shmouni F, Verbalis JG, Koch CA. Hypophysitis: An update on the novel forms, diagnosis and management of disorders of pituitary inflammation. Best Pract Res Clin Endocrinol Metab. 2019;33(6):101371.

2. Refardt J, Winzeler B, Christ-Crain M. Copeptin and its role in the diagnosis of diabetes insipidus and the syndrome of inappropriate antidiuresis. Clin Endocrinol (Oxf). 2019;91(1):22-32.

3. Fenske W, Allolio B. Current state and future perspectives in the diagno sis of diabetes insipidus: A clinical review. J Clin Endocrinol Metabolism. 2012;97(10):3426-37.

4. Honegger J, Schlaffer S, Menzel C, Droste M, Werner S, Elbelt U, et al.; Pituitary Working Group of the German Society of Endocrinology. Diagnosis of primary hypophysitis in Germany. J Clin Endocrinol Metab. 2015;100(10):3841-9.

5. Pérez $G$, Almeda-Valdés $P$, Cuevas-Ramos $D$, Juárez-Comboni SC, Higuera-Calleja J, Gómez-Pérez FJ. Hipofisitis autoinmune. Serie de casos y revisión de la literatura. Gac Med Mex. 2013;149(2): 229-36.

6. Gutenberg A, Larsen J, Lupi I, Rohde V, Caturegli P. A radiologic score to distinguish autoimmune hypophysitis from nonsecreting pituitary adenoma preoperatively. AJNR Am J Neuroradiol. 2009;30(9): 1766-72.

7. Caranci F, Leone G, Ponsiglione A, Muto M, Tortora F, Muto M, et al. Imaging findings in hypophysitis: a review. Radiol Med. 2020;125(3): 319-28.

8. Prete A, Salvatori R. Hypophysitis. En: Feingold KR, Anawalt B, Boyce A, et al., editores [Internet]. South Dartmouth (MA): MDText.com, Endotext 2000 [fecha actualización: 15 de agosto de 2018]. Disponible en: https:// www.ncbi.nlm.nih.gov/books/NBK519842/?report=classic 